Measuring x-ray burn history with the Streaked Polar Instrumentation for Diagnosing Energetic Radiation (SPIDER) at the National Ignition Facility (NIF)

S. F. Khan, P. M. Bell, D. K. Bradley, S. R. Burns, J. R. Celeste, L. S. Dauffy, M. J. Eckart, M. Geissel, D. I. Headley, J. P. Holder, N. Izumi, M. C. Jones, J. W. Kellogg, H. Y. Khater, J. R. Kimbrough, A. G. MacPhee, Y. P. Opachich, N. E. Palmer, R. B. Petre, J. L. Porter, R. T. Shelton, T. L. Thomas, J. B. Worden

July 25, 2012

SPIE Optics and Photonics 2012: Optical Engineering + Applications

San Diego, CA, United States August 12, 2012 through August 14, 2012 
This document was prepared as an account of work sponsored by an agency of the United States government. Neither the United States government nor Lawrence Livermore National Security, LLC, nor any of their employees makes any warranty, expressed or implied, or assumes any legal liability or responsibility for the accuracy, completeness, or usefulness of any information, apparatus, product, or process disclosed, or represents that its use would not infringe privately owned rights. Reference herein to any specific commercial product, process, or service by trade name, trademark, manufacturer, or otherwise does not necessarily constitute or imply its endorsement, recommendation, or favoring by the United States government or Lawrence Livermore National Security, LLC. The views and opinions of authors expressed herein do not necessarily state or reflect those of the United States government or Lawrence Livermore National Security, LLC, and shall not be used for advertising or product endorsement purposes. 


\title{
Measuring x-ray burn history with the Streaked Polar Instrumentation for Diagnosing Energetic Radiation (SPIDER) at the National Ignition Facility (NIF)
}

\author{
S. F. Khan*a, P. M. Bell ${ }^{\mathrm{a}}$, D. K. Bradley ${ }^{\mathrm{a}}$, S. R. Burns ${ }^{\mathrm{a}}$, J. R. Celeste $^{\mathrm{a}}$, L. S. Dauffy ${ }^{\mathrm{a}}$, M. J. Eckart ${ }^{\mathrm{a}}$, \\ M. A. Gerhard ${ }^{a}$, C. Hagmann ${ }^{a}$, D. I. Headley ${ }^{b}$, J. P. Holder ${ }^{a}$, N. Izumi ${ }^{a}$, M. C. Jones ${ }^{b}$, J. W. Kellogg ${ }^{b}$, \\ H.Y. Khater ${ }^{\mathrm{a}}$, J. R. Kimbrough ${ }^{\mathrm{a}}$, A. G. MacPhee ${ }^{\mathrm{a}}$, Y. P. Opachich ${ }^{\mathrm{a}}$, N. E. Palmer ${ }^{\mathrm{a}}$, R. B. Petre ${ }^{\mathrm{a}}$, J. L.

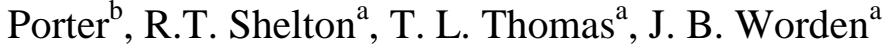 \\ ${ }^{a}$ Lawrence Livermore National Laboratory, 7000 East Ave, Livermore, CA, USA 94550 \\ ${ }^{\mathrm{b}}$ Sandia National Laboratories, 1515 Eubank Blvd. SE, Albuquerque, NM, USA 87123
}

\begin{abstract}
We present a new diagnostic for the National Ignition Facility (NIF) [1,2]. The Streaked Polar Instrumentation for Diagnosing Energetic Radiation (SPIDER) is an X-ray streak camera for use on almost-igniting targets, up to $\sim 10^{17}$ neutrons per shot. It measures the $\mathrm{X}$-ray burn history for ignition campaigns with the following requirements: X-Ray Energy 8-30keV, Temporal Resolution 10ps, Absolute Timing Resolution 30ps, Neutron Yield: $10^{14}$ to $10^{17}$. The features of the design are a heavily shielded instrument enclosure outside the target chamber, remote location of the neutron and EMP sensitive components, a precise laser pulse comb fiducial timing system and fast streaking electronics. SPIDER has been characterized for sweep linearity, dynamic range, temporal and spatial resolution. Preliminary DT implosion data shows the functionality of the instrument and provides an illustration of the method of burn history extraction.
\end{abstract}

Keywords: National Ignition Facility (NIF), inertial confinement fusion (ICF), streak camera, x-ray diagnostics, neutron mitigation, SPIDER, bang time, burn width

\section{INTRODUCTION}

Measurement of the absolute time (bang time) and burn width of the peak x-ray emission from the imploding cores of deuterium-tritium capsules near peak compression is integral to the success of the ignition campaign [3,4]. These measurements are used to validate implosion modeling and confirm the symmetry tuning parameters of the laser as well as the DT capsule. The challenge for x-ray diagnostics in inertial confinement fusion (ICF) experiments is to not only withstand the damage from an intense flux of energetic neutrons, but also to minimize the detrimental signal contribution from these neutrons, electromagnetic impulses and background x-rays. The SPIDER was specifically designed to measure the $\mathrm{x}$-ray bang time and burn width in a neutron yield of up to $5 \times 10^{16}$ at target chamber center (TCC). It manages to achieve this by enclosing the instrument in heavy shielding, positioning the instrument several meters away outside the target chamber, and temporally gating the micro-channel plate.

SPIDER has recently been commissioned at NIF and has been run on several shots. Preliminary implosion data is very promising and clearly illustrates the capabilities of the SPIDER. The next section describes the design and architecture of the instrument. Section three reports the performance of SPIDER as determined by a series of standardized calibration procedures. Section four defines the process of extracting the implosion signal from the hohlraum emission background.

*khan9@1lnl.gov 


\section{DESIGN}

The architecture of the SPIDER is motivated by neutron mitigation while maintaining high precision. The drawings of the general arrangement of the instrument (figures 1 and 2) emphasizes the additional shielding, line of sight (LOS) of the instrument, the distance of the instrument from TCC, and the separate vacuum system. The platform holding the SPIDER cart is composed of two 1" steel plates and 12" of high-density polyethylene (HDPE) in between the plates. Additionally, there is an air gap in the beam line from the LOS tube to the SPIDER cart. The LOS tube is capped with a $5 \mathrm{~mm}$ thick polyimide window which prevents the target chamber from venting due to debris hitting the window. Unfortunately, this greatly reduces the instruments response for $\mathrm{x}$-rays below $10 \mathrm{keV}$. There is a plan to replace this with an appropriately thick beryllium window once beryllium is allowed for use in the target chamber. One of the most important features of SPIDER is the $30^{\circ}$ angle of incidence of the LOS with respect to the axis of the instrument; it prevents neutrons and hard x-ray signals from directly interacting with the CCD and streak tube. Another essential aspect of the instrument is the long distance from the slit to the TCC. At $~ 9200 \mathrm{~mm}$, the difference between the time of flight between $\mathrm{x}$-rays and $14 \mathrm{MeV}$ neutrons is about $150 \mathrm{~ns}$, which ensures that the neutron signal does not interfere with the x-ray implosion emission signal. The field of view of SPIDER is a circle with a diameter of about $150 \mathrm{~mm}$ at TCC.

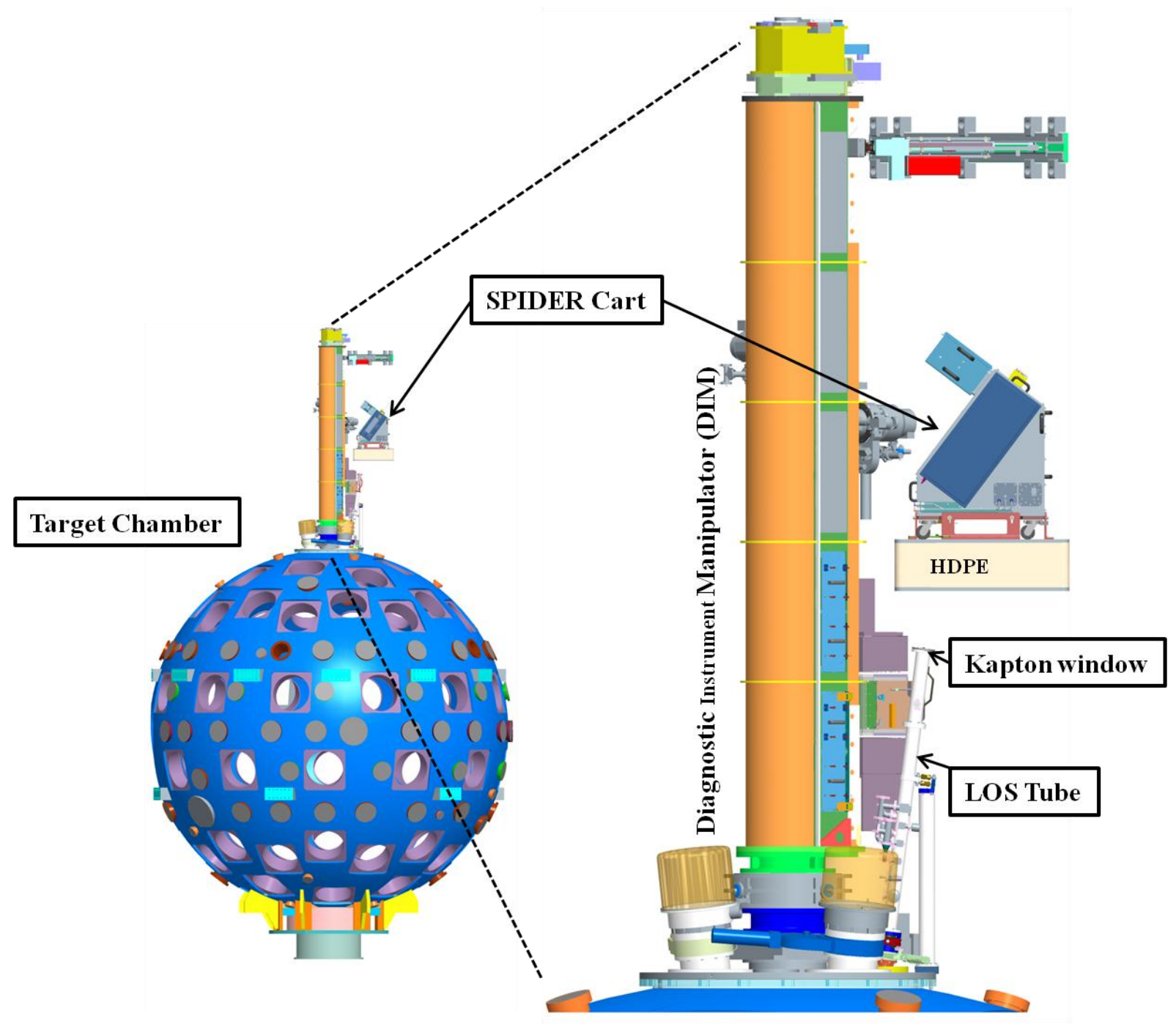

Figure 1. Model of the NIF target chamber, Polar Diagnostic Instrument Manipulator (DIM), SPIDER cart, and LOS tube. Note the large distance between SPIDER and TCC $(\sim 9235 \mathrm{~mm})$. The platform holding SPIDER is composed of two 1" steel plates and 12" of high-density polyethylene (HDPE). The SPIDER axis is at a $30^{\circ}$ angle of incidence with respect to the LOS. The field of view as seen by SPIDER at TCC is $\sim 150 \mathrm{~mm}$ wide. 
Figure 2, below, shows the SPIDER cart in detail. The cart has an independent vacuum pumping system and there is a $1 \mathrm{~mm}$ polyimide window for the $\mathrm{x}$-ray signal. This design isolates the target chamber vacuum system from that of the SPIDER. Several inches of tungsten line the front and sides of the housing to shield the instrument from neutrons and gamma rays. Thick HDPE material is used as additional neutron shielding and placed on the bottom and sides of the cart. A set of filters allows for the study of the spectral properties of the x-ray emission. The current design uses three filters: $33 \mu \mathrm{m}$ Zinc, $14 \mu \mathrm{m}$ Aluminum and $11 \mu \mathrm{m}$ Niobium. The resulting recorded filtered SPIDER signal is shown in figure 6.

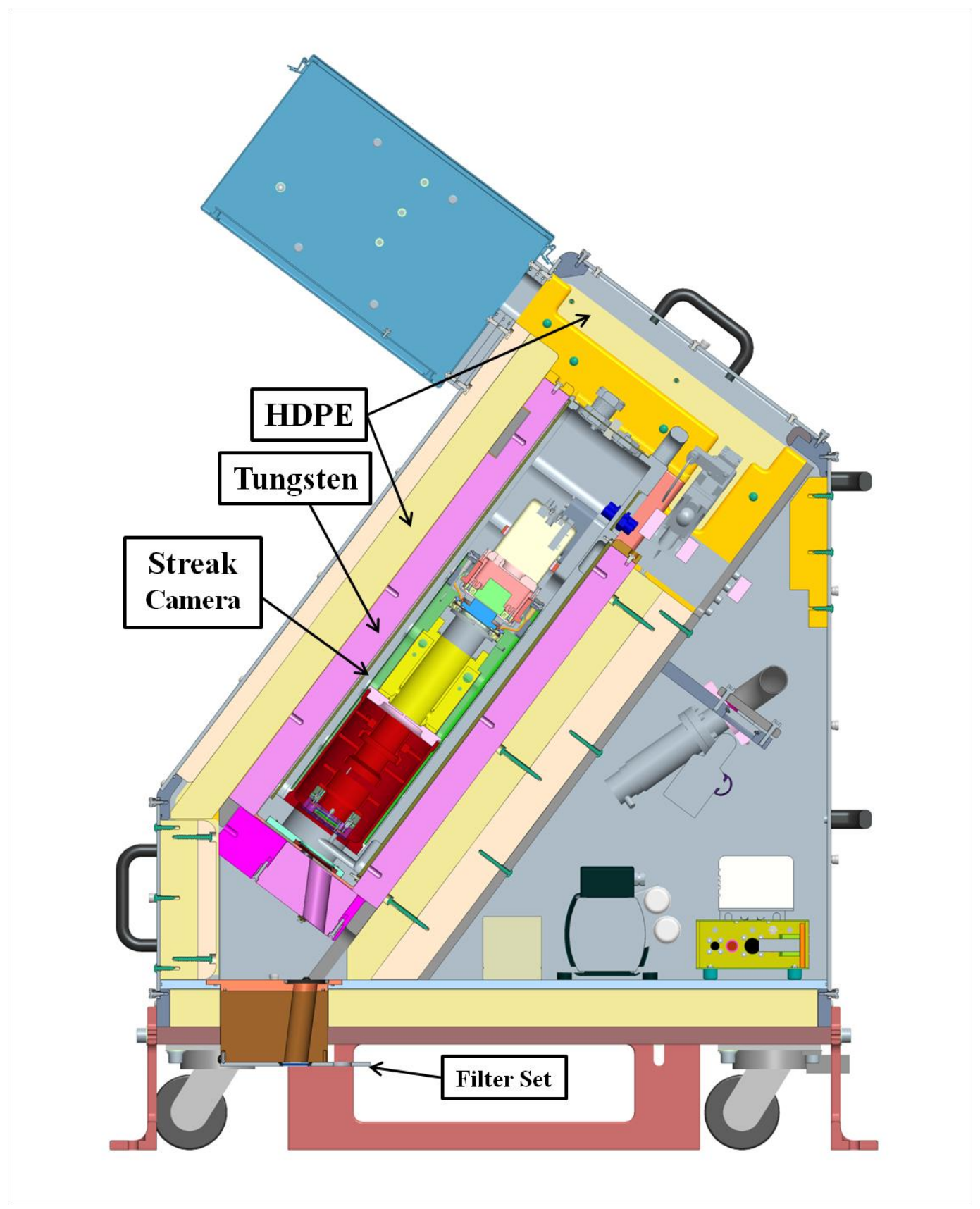

Figure 2. Cross-section of the SPIDER cart showing the shielding employed. Thick Tungsten metal (pink colored) surrounds the front and sides of the aluminum housing followed by high-density polyethylene (cream colored). 
The SPIDER recording system is built inside an aluminum housing similar to the DIM Imaging Streak Camera (DISC) $[5,6]$ currently fielded at the NIF. Figure 3 shows the main components of the SPIDER: the photocathode/slit assembly, the Kentech supplied electron optics and sweep assembly, the image intensifier assembly (micro-channel plate/phosphor), and the CCD camera. The photocathode assembly consists of a $250 \mu \mathrm{m}$ slit that is angled by $30^{\circ}$ about the horizontal diagonal of the beam path so that any direct LOS x-ray signal is not imaged by the phosphor and CCD camera. The photocathode consists of two materials deposited side by side onto a $1000 \AA$ Lexan substrate: x-rays are converted to photoelectrons with $2000 \AA$ of CsI that is deposited onto a $300 \AA$ Au conductive layer, the other section utilizes $500 \AA$ of $\mathrm{Al}$ which is devoted to converting the $263 \mathrm{~nm}$ fiducial comb pulses into electrons [6]. The final component of the photocathode assembly is a mesh, used to apply a high voltage gradient needed to accelerate the photoelectrons past a set of electrostatic focusing lenses and deflection plates onto the intensifier assembly. The focusing lenses introduce a 1.2-1.3X internal magnification. The sweep assembly consists of a pair of deflection plates driven by a $\sim 1.2 \mathrm{kV}$ voltage ramp. A bias voltage can be applied to either plate in between $-1 \mathrm{kV}$ to $1 \mathrm{kV}$ in order to adjust the position of the swept image in the focal plane. The image intensifier assembly consists of a MCP coupled to a phosphor coated fiber optic face plate. The MCP pores have a length to diameter ratio (L/D) of 46:1, an open area ratio of $60 \%$ and a bias angle of $8^{\circ}$. There are three possible MCP gain settings: Low $\sim 650 \mathrm{~V}$, Medium $\sim 750 \mathrm{~V}$, and High $\sim 850 \mathrm{~V}$. The usual setting is medium due to a good balance of signal to noise and dynamic range of the instrument. The phosphor coating on the $40 \mathrm{~mm}$ length fiber optic faceplate is the P43 type, which produces $545 \mathrm{~nm}$ light. The downside to this phosphor type is the slow extinction of about $1.5 \mathrm{~ms}$. In order to reduce the contribution of the neutron background signal, the MCP and gating electronics were built with a fast shunt to ground of about 10ns. The CCD

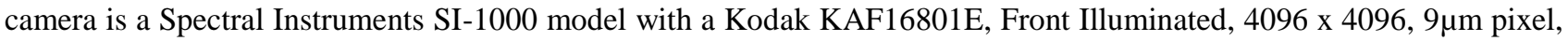
full frame CCD [7]. The CCD is thermoelectrically cooled to $-20^{\circ} \mathrm{C}$ which nominally produces dark current at a rate of $0.05 \mathrm{e}-/ \mathrm{pixel} / \mathrm{s}$.

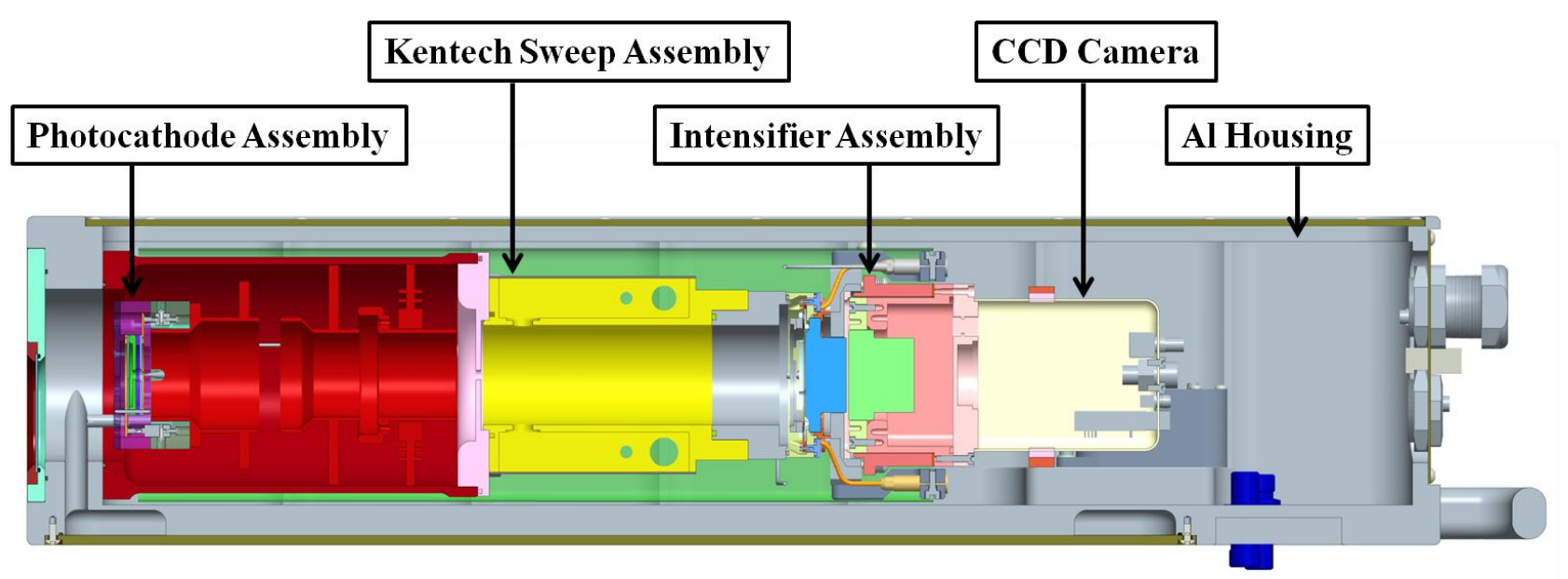

Figure 3. The main components of the SPIDER are shown as situated inside the aluminum housing. From left to right: the photocathode and slit assembly, the Kentech streak tube assembly, the image intensifier assembly, and the CCD camera.

In order to precisely measure the bang time (BT) and burn width (BW) of the capsule implosion, a comb of $45 \mathrm{ps}$ FWHM pulses are used to determine the time scale of the x-ray streak image [8]. The pulse comb appears as a series of six spots on the left edge of the image that spans $1.5 \mathrm{~ns}$. The pulses are produced by siphoning a small percentage of the NIF seed laser, then splitting the pulse with a fiber optic splitter, then temporally delaying each pulse by sending the pulse through different length fiber optic cables [6,9]. The pulses are frequency quadrupled to $263 \mathrm{~nm}$ using two second harmonic crystals. This frequency conversion along with further amplification occurs inside a separate aluminum housing that is attached to the SPIDER cart (not shown). The fiducial system has been successfully tested with SPIDER off line and implemented on DISC, illustrating an absolute timing accuracy of 4-5ps. 


\section{PERFORMANCE CHARACTERISTICS}

\subsection{Calibration}

Before fielding the instrument, the performance and characteristics of the instrument were determined by a series of measurements and calibrations. The characterization method is the standardized procedure used for the DISC and other streak cameras at NIF $[10,11]$, and measures the spatial resolution, temporal resolution, dynamic range, sweep linearity, and spatial uniformity (flat-field) of SPIDER. A summary of the results of this characterization is presented in Table 1 for the sweep settings we will use: $1.5 \mathrm{~ns}, 2 \mathrm{~ns}, 3 \mathrm{~ns}$, 5ns. For each sweep window setting, the actual sweep window depends on the detailed as-built streak electronics and the actual separation from the deflector plates and the image intensifier. The spatial resolution is a measure of the minimum feature that can be distinguished in the spatial direction. The $1.5 \mathrm{~ns}$ setting produced low counts which accounts for the larger value and uncertainty. The temporal resolution is determined by the size of the slit in front of the photocathode and shortens for smaller sweep windows. The $5^{\text {th }}$ column shows the level of CCD counts where a pulse shorter than the temporal resolution broadens by $20 \%$. Since this effect is mostly dependent on the space charge repulsion in the focusing part of the tube and not on the sweeping part, the levels for the different sweep settings are very close. The sweep linearity and flat-field data cannot be shown in tabular form, but examples of these are given in ref [10,12]. Warping of the image arises from the cylindrical electron optics between the photocathode and the image intensifier. This effect from the electron optics is for the most part, reproducible and correctable. The sweep linearity is measured in order to develop a time history correction to the streak image. The spatial uniformity is measured by taking a slow sweep across the CCD with a uniform flat in time $\mathrm{x}$-ray source. This test allows for the creation of a flat-field correction image that mitigates the contribution from non-uniformities in the MCP and phosphor coated fiber optic face plate.

Table 1. Measured characteristics of the SPIDER.

\begin{tabular}{|c|c|c|c|c|}
\hline $\begin{array}{c}\text { Sweep Speed } \\
\text { Setting, ns }\end{array}$ & $\begin{array}{c}\text { Measured sweep } \\
\text { window, ns }\end{array}$ & $\begin{array}{c}\text { Spatial } \\
\text { Resolution, } \boldsymbol{\mu m}\end{array}$ & $\begin{array}{c}\text { Temporal } \\
\text { Resolution, ps }\end{array}$ & $\begin{array}{c}\text { Broadening Limit (medium } \\
\text { gain), CCD counts }\end{array}$ \\
\hline 1.5 & 1.345 & $139 \pm 37$ & 8.1 & $3380 \pm 500$ \\
\hline 2 & 1.849 & $98 \pm 4$ & 12.6 & $3800 \pm 500$ \\
\hline 3 & 2.626 & $104 \pm 12$ & 19.1 & $4200 \pm 500$ \\
\hline 5 & 4.542 & $109 \pm 24$ & 34.6 & $3600 \pm 500$ \\
\hline
\end{tabular}

\subsection{Neutron Effects}

The effects of neutrons on the SPIDER were carefully investigated in order to design adequate shielding and predict the neutron yield limits for which the instrument can continue to measure the $\mathrm{x}$-ray burn history within the specified tolerance. Simulations of the neutron and photon flux at both the photocathode and the CCD were done using the general Monte Carlo N-Particle (MCNP) transport code [13]. Figure 4 shows plots of the simulated neutron (left plot) and the gamma ray (right plot) per $\mathrm{MeV}$ per source neutron at the CCD position for two cases: no SPIDER shielding and current SPIDER shielding configuration. The simulations show that the current shielding configuration reduces the 14 $\mathrm{MeV}$ neutron flux by a factor of 33 and the integrated neutron flux by 25 . The gamma ray contribution is reduced by an order of magnitude. The CCD degradation due to saturated pixels is proportional to the amount of energy deposited in the silicon. For a neutron yield of $10^{16}$, the integrated deposited energy with shielding is calculated to be $0.0045 \operatorname{rad}(\mathrm{Si})$ from neutrons and $0.025 \mathrm{rad}(\mathrm{Si})$ from gamma rays. Figure 5 shows the simulated images [14] of an ideal capsule emission with neutron background noise: $10^{16}$ neutron yield (left) and $10^{17}$ neutron yield (right). The simulated images imply that with the current shielding configuration, SPIDER will still be able to report accurate BW and BT values. In the $10^{16}$ neutron yield case the signal to noise ratio $(\mathrm{S} / \mathrm{N})$ is $\sim 7.5$ whereas in the $10^{17}$ neutron yield case the $\mathrm{S} / \mathrm{N}$ is $\sim 0.7$. Averaging over all the rows of data will significantly reduce the effects of the high energy radiation induced background. Measurement of the BT and BW on the noisy data and comparing with the clean simulation indicates that the neutroninduced noise contribution will generate an uncertainty of the measurement of the BT \& BW of $\sim 5 \mathrm{ps}$. 

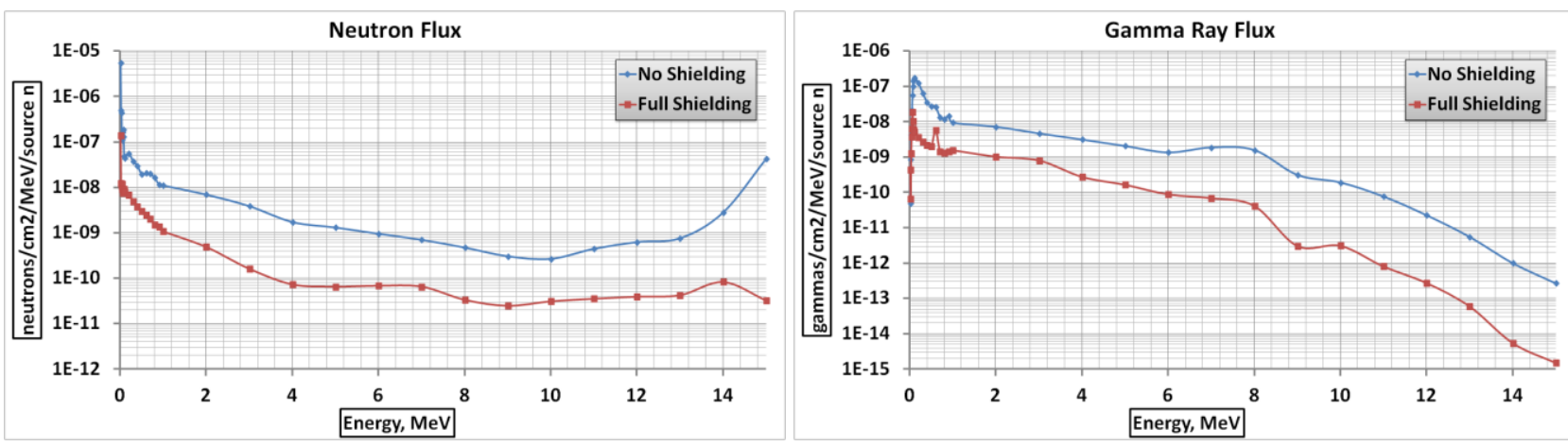

Figure 4. Left: Plot of simulated neutron per MeV per source neutron at the CCD position with and without the current SPIDER shielding. Right: Plot of simulated gamma ray per $\mathrm{MeV}$ per source neutron at the CCD position with and without the current SPIDER shielding. The simulations were done using the MCNP code. The shielding reduces the integrated neutron flux by a factor of $\sim 25$.
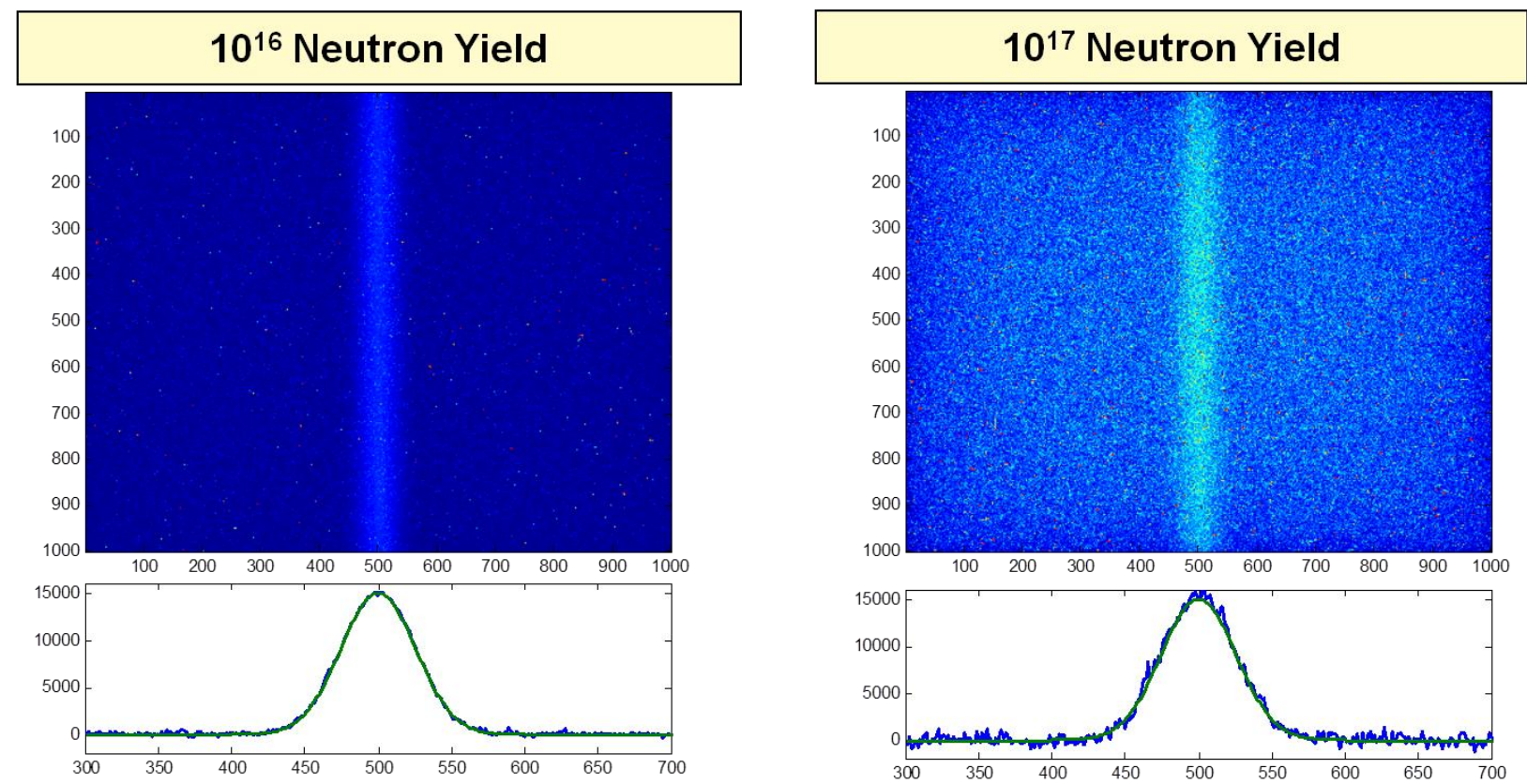

Figure 5. Simulated images of capsule emission with neutron background noise for $10^{16}$ neutron yield on the left and $10^{17}$ neutron yield on the right. The x-ray emission is scaled to what is expected for these yield levels.

\section{INITIAL RESULTS FROM THE NIF}

A series of recent implosion experiments exemplifies the capabilities of SPIDER. The instrument sees both the x-ray emission from the hohlraum as well as that from the imploding capsule. Figure 6 shows the streak image from a recent implosion experiment at the NIF. Time flows from bottom to top and the total duration of the streak on this image is $\sim 4.5 \mathrm{~ns}$. In order to extract the BW and BT of the implosion, the following procedure is used. First, the hohlraum emission is modeled by an exponentially decaying function of the form 


$$
a_{0}+b_{0} e^{-\frac{t-b_{1}}{b_{3}}}+c_{0} e^{-\frac{t-c_{1}}{c_{3}}}
$$

After removing the hohlraum emission contribution from the signal, the remaining signal is fitted to a Gaussian function in order to retrieve the width and offset. These steps are illustrated in Figure 7. In this particular DT shot, SPIDER measured an x-ray burn width of $\sim 168 \pm 12 \mathrm{ps}$ and a bang time of $\sim 24.17 \pm 0.022 \mathrm{~ns}$. These values are within the range of results reported by the other $\mathrm{x}$-ray diagnostic instruments that reports BT and/or BW.

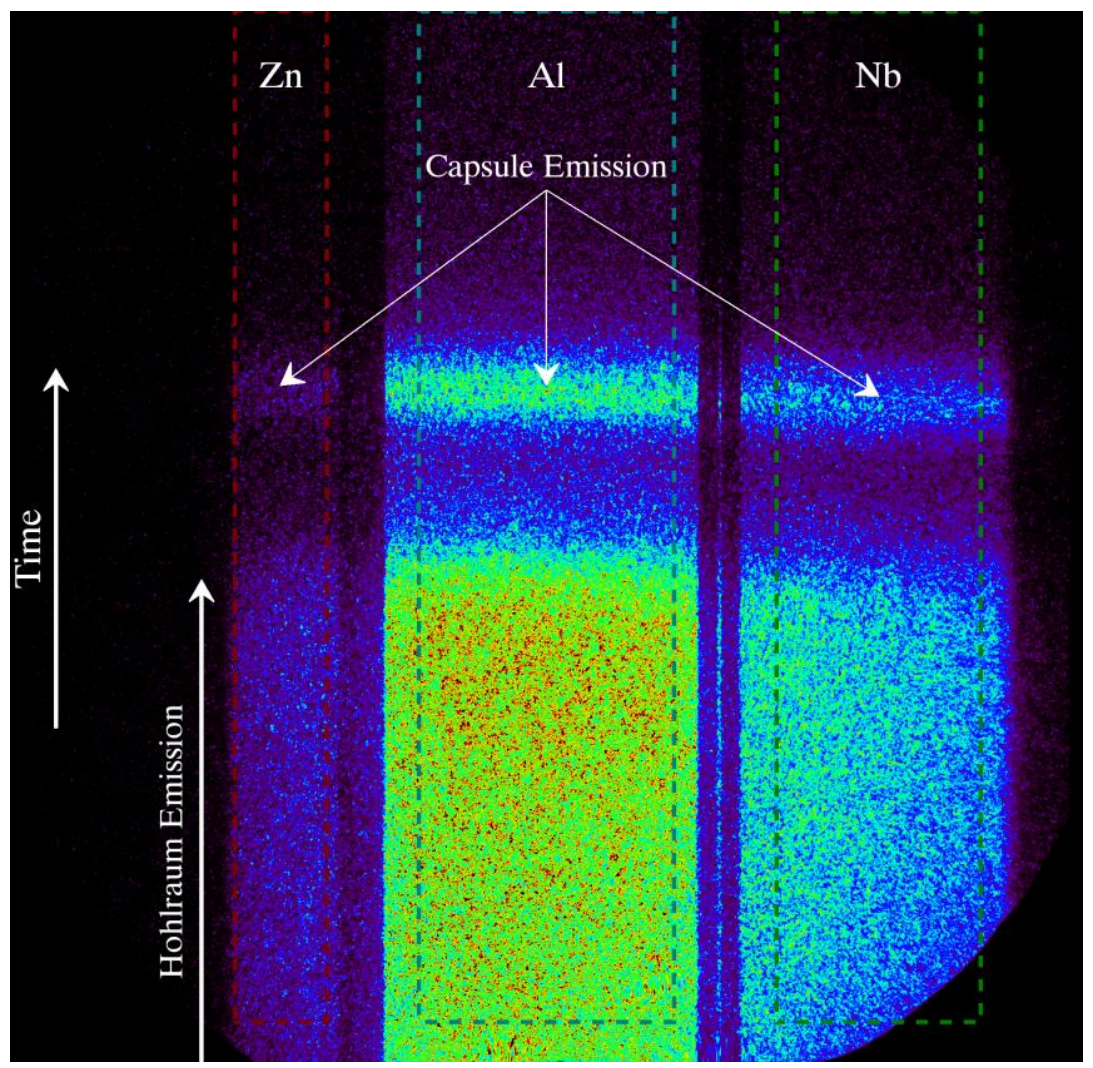

Figure 6. The streak image from SPIDER shows the hohlraum emission and the capsule emission. Time flows from bottom to top and the streak duration on the camera is $4.54 \mathrm{~ns}$ long. The SPIDER was fielded with a set of three filters for spectral binning: $33 \mu \mathrm{m}$ Zinc, $14 \mu \mathrm{m}$ Aluminum and $11 \mu \mathrm{m}$ Niobium.
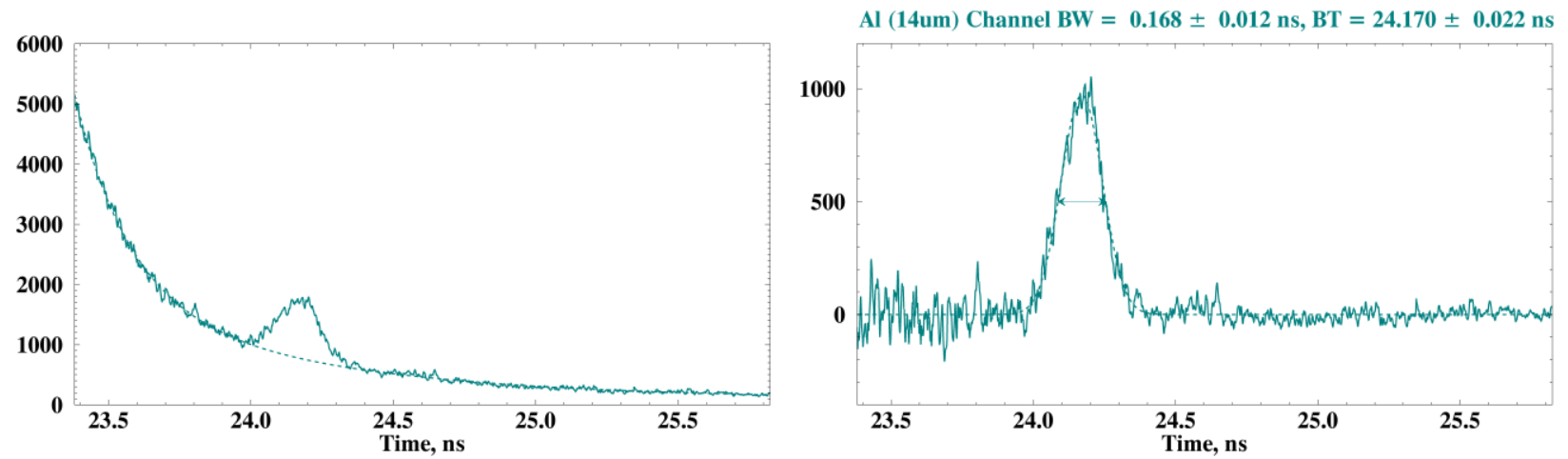

Figure 7. Left: Plot of the average of the columns within the Al filter. The dashed line indicates the exponential fit. Right: Gaussian fit of the extracted capsule emission showing a resulting burn width of $\sim 168 \mathrm{ps}$ and a bang time of $\sim 24.17 \mathrm{~ns}$. 


\section{CONCLUSION}

SPIDER is a high resolution burn history diagnostic that has been fielded on implosion shots at the NIF. The SPIDER was built to operate in a high TCC neutron environment of $5 \times 10^{16}$ into $4 \pi \mathrm{r}^{2}$. This was accomplished by heavy shielding, complex electronics and an integrated design. Simulations of the effects of neutrons and gamma rays on SPIDER determined the shielding configuration and set the operating limits for reporting burn width and bang time within specifications. Characterization of the instrument showed that the instrument performed within the temporal resolution specifications. The sweep linearity and the flat field measurements provided correction data that are currently applied to the NIF experimental data. A simple signal extraction technique is employed in order to report the burn width and bang

time of the imploding capsule. Preliminary results indicate that the instrument is capable of recording the burn history of imploding capsules with the accuracy required by the ignition campaign.

\section{ACKNOWLEDGEMENT}

This work was performed under the auspices of the U.S. Department of Energy by Lawrence Livermore National Laboratory under contract DE-AC52-07NA27344 and by Sandia National Laboratories under contract DE-AC0494AL85000. LLNL-CONF-566492

\section{REFERENCES}

[1] Lindl, J. D., Amendt, P., Berger, R. L., Glendinning, S. G., Glenzer, S. H., Haan, S. W., Kauffman, R. L., Landen, O. L., and Suter, L. J., "The physics basis for ignition using indirect-drive targets on the National Ignition Facility," Phys. Plasmas 11, 339 (2004)

[2] Glenzer, S. H., Callahan, D. A., MacKinnon, A. J., Kline, J. L., Grim, G., et al., "Cryogenic thermonuclear fuel implosions on the National Ignition Facility," Phys. Plasmas 19, 056318 (2012)

[3] Meezan, N. B., Atherton, L. J., Callahan, D. A., Dewald, E. L., Dixit, S., Dzenitis, E. G., Edwards, M. J., Haynam, C. A., Hinkel, D. E., Jones, O. S., Landen, O., London, R. A., Michel, P. A., Moody, J. D., Milovich, J. L., Schneider, M. B., Thomas, C. A., Town, R. P. J., Warrick, A. L., Weber, S. V., Widmann, K., Glenzer, S. H., Suter, L. J., MacGowan, B. J., Kline, J. L., Kyrala, G. A., and Nikroo, A., "National Ignition Campaign Hohlraum energetic," Phys. Plasmas 17, 056304 (2010)

[4] Landen, O. L., et al., "Capsule performance optimization in the National Ignition Campaign," Phys. Plasmas 17, $056301(2010)$

[5] Kimbrough, J. R., Bell, P. M., Bradley, D. K., Holder, J. P., Kalantar, D. H., MacPhee, A. G., Telford, S., "Standard design for National Ignition Facility x-ray streak and framing cameras," Rev. Sci. Instr. 81(10), 10E530 (2010)

[6] Opachich, Y.P., Palmer. N., Homoelle. D., Hatch, B., Bell, P.M., Bradley, D. K., Kalantar, D. H., Browning, D., Zuegel, J., and Landen, O. L., "X-ray Streak Camera Cathode Development and Timing Accuracy of the 4w UV Fiducial System at the National Ignition Facility," Rev. Sci. Instr. 83(10), 10E123 (2012)

[7] Kimbrough, J. R., Moody, J. D., Bell, P. M., Landen, O. L., "Characterization of the Series 1000 Camera System,” Rev. Sci. Instr. 75, 4060 (2004)

[8] Jaanimagi, P. A., DaSilva, L., Gregory, G. G., Hestdalen, C., Kiikka, C. D., Kotmel, R., Richardson, M. C., "Optical fiducials for x-ray streak cameras at LLE," Rev. Sci. Instr. 57(8), 2189 (1986)

[9] Homoelle, D., "A compact UV timing fiducial system for use with x-ray streak cameras at the NIF," These proceedings, 2012

[10] Charest, M. R., Torres, P., Silbernagel, C. T., Kalantar, D. H., "Reliable and repeatable characterization of optical streak cameras," Rev. Sci. Instr. 79(10), 10F546 (2008)

[11] Kalantar, D. H., Bell, P. M., Costa, R. L., Hammel, B. A., Landen, O. L., Orzechowski, T. J., Hares, J., and Dymoke-Bradshaw, A. K., "Characterization of x-ray streak cameras for use on Nova," Proc. SPIE 2869, 680 (1997)

[12] Silbernagel, C. T., Torres, P., Kalantar, D. H., "A method for analyzing high-resolution time-domain streak camera calibration data," Advanced Signal Processing Algorithms, Architectures, and Implementations XIV, 435-442 (2004)

[13] "MCNP - A General Monte Carlo N-Particle Transport Code, Version 5," Los Alamos National Laboratory, LAUR-03-1987 (2005) 
[14] Hagmann, C., Izumi, N., Bell, P., Bradley, D., Conder, A., Eckart, M., Khater, H., Koch, J., Moody, J., and Stone, G., "Modeling of neutron induced backgrounds in X-ray framing cameras," Rev. Sci. Instr. 81, 10E514 (2010) 